\title{
TRAJETÓRIA PROFISSIONAL DE TREINADORES NO CONTEXTO DO ESPORTE PARALÍMPICO
}

\author{
PROFESSIONAL TRAJECTORY OF COACHES IN THE CONTEXT OF \\ PARALYMPIC SPORTS
}

\section{TRAJETORIA PROFESIONAL DE ENTRENADORES EN EL CONTEXTO DEL DEPORTE PARALÍMPICO}

\author{
Lucas Alves Facundo*, Marco Túlio de Mello*, Mário Antônio de Moura Simim**, \\ Tiago Duarte ${ }^{* * *}$, Aline Ângela da Silva Cruz ${ }^{\star}$, Fernanda Veruska Narciso*, \\ Roberta Ariane de Andrade Ramos*, Alberto Martins da Costa ${ }^{* *+*}$, \\ Andressa Silva*
} Andressa Silva*

\begin{abstract}
Palavras chave: Esportes para pessoas com deficiência. Capacitação profissional. Educação Física e treinamento.

Resumo: Treinadores do esporte paralímpico apresentam diferenças na atuação comparados a outros treinadores. Assim, o objetivo do presente estudo foi analisar a trajetória profissional dos treinadores da Delegação Paralímpica Brasileira dos Jogos Rio 2016. Este estudo contou com 35 treinadores (idade: $39,7 \pm 9,4$ anos, tempo de atuação: $10,7 \pm 5,4$ anos) que responderam a um questionário estruturado com perguntas fechadas relacionadas à sua trajetória e formação profissional. Em relação à formação acadêmica, $97,1 \%$ dos treinadores apresentaram graduação em Educação Física, 65,7\% cursaram pós-graduação lato sensu e 22,9\% pós-graduação stricto sensu (mestrado) na área. Além disso, $65,7 \%$ dos treinadores realizaram alguma disciplina na universidade relacionada ao esporte paralímpico e $85 \%$ realizaram cursos de formação complementar. Concluimos que os treinadores tiveram oportunidades de aprendizado formal durante a graduação em Educação Física e em outros cursos de formação após a conclusão do curso, bem como oportunidades informais para construírem sua carreira no esporte paralímpico.
\end{abstract}

Keywords: Sports for Persons with Disabilities. Professional Training. Physical Education and Training.

Palabras clave: Deportes para personas con discapacidad. Capacitación profesional. Educación Física y entrenamiento.

Abstract: Paralympic sports coaches work differently from other coaches. This study analyzed the professional trajectory of coaches working with the Brazilian Paralympic Delegation to the Rio 2016 Olympic Games. It included 35 coaches (age: $39.7 \pm 9.4$; working experience: $10.7 \pm 5,4$ years) who answered a structured questionnaire with closed-ended questions related to their history and professional training. As for academic training, $97.1 \%$ held degrees in Physical Education; 65.7\% held specialization diplomas; and $22.9 \%$ held master's degrees in the area. In addition, $65.7 \%$ of the coaches attended some collegelevel course on Paralympic sports and $85 \%$ attended complementary training courses. We concluded that coaches had formal learning opportunities during their undergraduate training in Physical Education and other courses after that, as well as informal opportunities to build their career in Paralympic sports.

Resumen: Los entrenadores del deporte paralímpico presentan diferencias en su actuación en comparación a otros entrenadores. El objetivo del presente estudio fue analizar la trayectoria profesional de los entrenadores de la Delegación Paralímpica Brasileña de los Juegos Río 2016. Este estudio contó con 35 entrenadores (edad: $39,7 \pm 9,4$ años, tiempo de actuación: $10,7 \pm 5,4$ años) que respondieron a un cuestionario estructurado con preguntas relacionadas a su trayectoria y formación profesional. En lo referente a formación académica, el $97,1 \%$ de los entrenadores eran graduados en Educación Física, 65,7\% cursó postgrado lato sensu y 22,9\% posgrado strito sensu en el área. Además, $65,7 \%$ de los entrenadores realizaron alguna disciplina en la universidad relacionada deporte paralímpico y el $85 \%$ realizaron cursos de formación complementaria. Concluimos que los entrenadores tuvieron oportunidades de aprendizaje formal durante su graduación en Educación Física y en otros cursos de formación después de la conclusión del curso, así como oportunidades informales para construir su carrera en el deporte paralímpico.
*Universidade Federal de Minas Gerais. Belo Horizonte, MG, Brasil. E-mail: lucasafac1996@gmail.com; tmello@demello.net.br; ninafisiobh@hotmail.com; fernandanarciso@ hotmail.com; roberta_andrade0612@hotmail.com; silvadressa@gmail.com

**Universidade Federal do Ceará. Fortaleza, CE, Brasil. E-mail: mams.ef@gmail.com

***University of Ottawa. Ottawa, Canadá.

E-mail: tiagofdd@gmail.com

****Comitê Paralímpico Brasileiro. São Paulo, SP, Brasil.

E-mail: amcosta@cpb.org.br

Recebido em: 01-10-2018 Aprovado em: 29-04-2019 Publicado em: 03-06-2019

DOI: https://doi.org/10.22456/1982-8918.87115

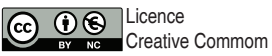




\section{INTRODUÇÃO}

No contexto esportivo, treinadores apresentam diversas funções no corpo técnico de suas equipes, tais como manutenção da coesão do grupo, estabelecimento de metas, melhoria do desempenho esportivo, além da formação de ambiente propício para treinamento de elite e construção de identidade competitiva em seus atletas (FALCÃO; BLOOM; HARVEY, 2015; PERRET, 2015; DOUGLAS;FALCÃO; BLOOM., 2018). No caso de treinadores no esporte paraolímpico, sua rotina de trabalho apresenta particularidades relacionadas ao processo de treinamento de atletas com deficiência. Assim, esses treinadores devem realizar adaptações em materiais e equipamentos utilizados pelos atletas (por exemplo, prótese ou cadeira de rodas) além de analisar a acessibilidade dos locais de treinamento, hospedagem e competição (TAYLOR; WERTHNET; CULVER, 2014; WAREHAM et al., 2017). No campo prático é comum treinadores auxiliando e orientando os atletas em tarefas do seu dia a dia não relacionadas ao ambiente esportivo (TAWSE et al., 2012). Todas essas razões contribuem para que esses profissionais se sobrecarreguem em funções extras, aumentando suas responsabilidades e comprometendo sua função principal.

Para que o treinador atinja seus objetivos e cumpra suas funções é necessário que ele seja eficaz e competente. Segundo Côté e Gilbert (2009), a eficácia do treinador passa por três componentes integrados: conhecimento do treinador, resultado dos atletas e contexto esportivo do treinador. 0 conhecimento do treinador relaciona-se com o conhecimento profissional, interpessoal e intrapessoal. O conhecimento profissional pauta-se no domínio técnico específico, no conhecimento curricular e pedagógico, indicando informações acerca da formação do treinador nas universidades, em cursos e workshops de aperfeiçoamento (CÔTÉ; GILBERT, 2009). Dessa forma, os conhecimentos necessários para atuar como treinador no esporte paralímpico englobam desde conhecimentos específicos a respeito das deficiências até questões relacionadas aos equipamentos utilizados na prática esportiva (CREGAN; BLOOM; REID, 2007; TAYLOR; WERTHNET; CULVER, 2014).

Nessa perspectiva, estudos buscaram compreender a trajetória dos treinadores no esporte paralímpico investigando como o contexto pessoal do treinador contribuiu para o sucesso esportivo nessa prática. Esses estudos reportam que parte do conhecimento específico desses treinadores foi obtido por meio de situações informais (DUARTE; CULVER, 2014; DOUGLAS; FALCÃO; BLOOM, 2018), tais como: busca de informações na internet, observações durante sessões de treinamentos, conversas com atletas e pais, interação com outros treinadores e profissionais da área (MCMASTER et al., 2012; DUARTE e CULVER, 2014; DOUGLAS; FALCÃO; BLOOM, 2018). Por outro lado, a dificuldade na obtenção de conhecimento específico a respeito do treinamento de atletas com deficiência em cursos e workshops tem sido apontada na literatura (MACDONALD et al., 2016). Adicionalmente, alguns autores (DUARTE e CULVER, 2014; TAYLOR et al., 2014) destacam a relevância da formação universitária em Educação Física e áreas afins como base para o início da carreira do treinador.

Pesquisas que investigam a trajetória de treinadores no esporte vêm contribuindo para indicar aspectos de necessidade na formação profissional (DUARTE; CULVER, 2014; DOUGLAS; FALCÃO; BLOOM, 2018). Embora o Brasil seja considerado potência no movimento paralímpico, estudos com treinadores brasileiros ainda são escassos. A partir dessa lacuna, 
pouco se sabe a respeito da trajetória desses profissionais. A literatura indica que dificuldades na formação de treinadores canadenses também existem, principalmente porque muitos cursos não conseguem suprir as suas necessidades de aprendizado (MCMASTER; CULVER; WERTHNER, 2012; MACDONALD et al., 2016). Em virtude disso e do crescimento das evidências científicas na área do esporte paralímpico, bem como da melhoria das condições de treinamento dos atletas e do elevado nível competitivo, conhecer a trajetória e formação dos treinadores emerge como questão evidente nos dias atuais. Para tanto, o objetivo do presente estudo foi analisar a trajetória profissional dos treinadores da Delegação Paralímpica Brasileira que atuaram nos Jogos Paralímpicos Rio 2016.

\section{PROCEDIMENTOS METODOLÓGICOS}

\subsection{TIPO DE ESTUDO}

O presente estudo é classificado como de caracterização, uma vez que esse tipo de pesquisa descreve fenômenos, mede e compara resultados de diferentes variáveis (VOLPATO, 2016).

\subsection{CUIDADOS ÉTICOS}

Este estudo foi aprovado pelo Comitê de Ética em Pesquisa da Universidade Federal de Minas Gerais, sob parecer 1.082 .55 e pela Academia Paralímpica Brasileira/Comitê Paralímpico Brasileiro. Foram garantidos aos treinadores a preservação de sua identidade e 0 anonimato na divulgação dos dados. Os voluntários consentiram sua participação na pesquisa de maneira online em formulário elaborado para essa finalidade.

\subsection{AMOSTRA}

Foram convidados todos os profissionais de Educação Física que participaram da Delegação Paralímpica Brasileira durante os Jogos Paralímpicos Rio 2016. Inicialmente, foram enviados e-mails para todos os 107 profissionais, dos quais 48 (44,9\%) responderam ao questionário estruturado disponibilizado online. Foram excluídos 13 indivíduos que não exercem a função de treinador. Desta maneira a amostra foi por conveniência, com participação de 35 treinadores (masculino: $n=34 ; 97,1 \%$ e feminino: $n=1 ; 2,9 \%$, idade de $39,7 \pm 9,4$ anos e tempo de atuação de 10,7 $\pm 5,4$ anos). Os treinadores atuaram em 21 modalidades paralímpicas, sendo alguns treinadores $(n=8 ; 22,8 \%)$ com atuação em mais de uma modalidade. A maioria dos treinadores atua em modalidades individuais ( $n=15 ; 71 \%$ ), em especial Atletismo e Natação ( $n=8 ; 17,0 \%$ em cada uma delas). A Tabela 1 apresenta a distribuição dos treinadores por modalidade paralímpica. Destacamos que 60\% $(n=21)$ dos treinadores apresentavam vínculo empregatício nestas modalidades, sendo que $31,4 \%(n=11)$ dos treinadores possuem com o Comitê Paralímpico Brasileiro. Adicionalmente, 57,1\% $(n=20)$ dos treinadores possuem medalhas em Jogos Paralímpicos e 71,4\% $(n=25)$ apresentam medalhas em Jogos Parapanamericanos. 
Tabela 1- Distribuição dos treinadores por modalidade paralímpica de atuação.

\begin{tabular}{lcc|lcc}
\hline Modalidades & $\mathbf{N}$ & $\%$ & Modalidades & $\mathbf{N}$ & $\%$ \\
\hline Atletismo & 8 & $17,0 \%$ & Goalball & 1 & $2,1 \%$ \\
Para-badminton & 1 & $2,1 \%$ & Halterofilismo & 1 & $2,1 \%$ \\
Basquete em Cadeira de Rodas (BCR) & 2 & $4,3 \%$ & Natação & 8 & $17,0 \%$ \\
Bocha & 3 & $6,4 \%$ & Rugby em Cadeira de Rodas (RCR) & 2 & $4,3 \%$ \\
Canoagem de Velocidade & 1 & $2,1 \%$ & Ski Cross Country & 1 & $2,1 \%$ \\
Ciclismo & 2 & $4,3 \%$ & Tênis de Mesa & 4 & $8,5 \%$ \\
Esgrima em Cadeira de Rodas (ECR) & 1 & $2,1 \%$ & Tênis em Cadeira de Rodas (TCR) & 3 & $6,4 \%$ \\
Futebol de 5 & 1 & $2,1 \%$ & Tiro com Arco & 2 & $4,3 \%$ \\
Futebol de 7 & 1 & $2,1 \%$ & Tiro Esportivo & 1 & $2,1 \%$ \\
Voleibol Sentado & 2 & $4,3 \%$ & Triátlon & 1 & $2,1 \%$ \\
Vela & 1 & $2,1 \%$ & Total & 47 & $100,0 \%$ \\
\hline
\end{tabular}

Fonte: Os autores

\subsection{ELABORAÇÃO DO INSTRUMENTO E COLETA DE DADOS}

O instrumento de pesquisa foi desenvolvido seguindo alguns passos necessários. Inicialmente foi realizada revisão de literatura para identificar possíveis instrumentos de pesquisa. A partir dessa fase, foram selecionados os estudos (CREGAN; BLOOM; REID, 2007; MCMASTER; CULVER; WERTHNER, 2012; DUARTE; CULVER, 2014; MACDONALD et al., 2016) que embasaram cientificamente as questões do instrumento de pesquisa.

A segunda etapa para construção do questionário foi encaminhá-lo para três professores experts na área de Educação Física Adaptada e Esporte Paralímpico. Segundo a literatura (ERICSSON, 2008; ERICSSON, 2014), o expert deve ter no mínimo dez mil horas ou dez anos de atuação em diferentes áreas do desenvolvimento humano e formação universitária condizente com a área de estudo. No presente estudo os experts são formados em Educação Física, com mais de dez anos de atuação no esporte paralímpico. Todos os experts já participaram de pelo menos uma competição internacional como membro de modalidades paralímpicas brasileiras. Nessa etapa todos experts analisaram as 63 questões do instrumento verificando se eram pertinentes aos objetivos do estudo. Após retorno dos experts foram realizadas alterações sugeridas e novamente enviamos o instrumento para eles, agora reduzido a 62 questões. Após terceira e última rodada de avaliação o questionário foi finalizado com 56 questões que obtiveram $80 \%$ de concordância entre os experts.

Foi utilizado como instrumento de coleta de dados um questionário online contendo 56 questões fechadas acerca da trajetória profissional dos treinadores, desta maneira sendo classificado como questionário estruturado. As questões do referido questionário são relacionadas à função do treinador junto à delegação, experiência no esporte adaptado/ paralímpico, modalidade esportiva em que atua, características demográficas e profissionais. O questionário foi inserido numa plataforma online (Google Formulários) visando facilitar o acesso e a participação de treinadores de todas as regiões do Brasil.

O link da plataforma com acesso ao questionário foi enviado por e-mail para todos os treinadores convocados pelo Comitê Paralímpico Brasileiro (CPB) para os Jogos Paralímpicos Rio 2016. Os e-mails dos treinadores foram obtidos junto à direção técnica do CPB. Cada treinador teve um período de três meses para responder o questionário (dezembro de $2016 \mathrm{a}$ 
fevereiro de 2017). Após a obtenção das respostas, os dados foram exportados para o software Microsoft Exce ${ }^{\circledR}$.

\subsection{TRATAMENTO DOS DADOS}

Para análise dos dados foi utilizada estatística descritiva composta por média \pm desviopadrão para dados contínuos e discretos e distribuição da frequência (absoluta e/ou relativa) para dados nominais e/ou ordinais.

\section{RESULTADOS}

Identificamos que 97,1\% ( $n=34)$ dos treinadores têm formação em Educação Física, com habilitação em Licenciatura ( $n=5 ; 14,3 \%)$, Bacharelado $(n=2 ; 5,7 \%)$ e Licenciatura/ Bacharelado ( $n=27 ; 77,1 \%$ ). Cabe ressaltar que somente um treinador não apresentou formação em Educação Física, sendo provisionado para atuar no Tiro Esportivo. 0 presente estudo também explorou o motivo do primeiro contato do treinador com o esporte paralímpico e pode-se observar que a maioria deles teve o primeiro contato durante o curso de graduação (Figura 1). Em relação aos dados da sua formação formal, que é o curso de graduação, pode-se observar que $65,7 \%(n=23)$ dos treinadores cursaram pelo menos uma disciplina relacionada à área de atividade física para pessoas com deficiência durante o curso de graduação. Adicionalmente a esse fato, $11,4 \%(n=4)$ dos treinadores cursaram mais de uma disciplina nesta área durante a graduação. As disciplinas realizadas pelos treinadores estão apresentadas na Tabela 2. Além disso, nossos resultados indicam que os treinadores graduados se formaram em instituições do ensino superior localizadas nas regiões: Sudeste (n $=24 ; 69 \%)$, Sul $(n=7 ; 20 \%)$, Centro-Oeste $(n=2 ; 6 \%)$ e Nordeste $(n=1 ; 3 \%)$, com apenas um treinador que se formou na região nordeste da Itália $(n=1 ; 3 \%)$.

Figura 1 - Primeiro contato dos treinadores com o esporte paralímpico $(n=24)$.

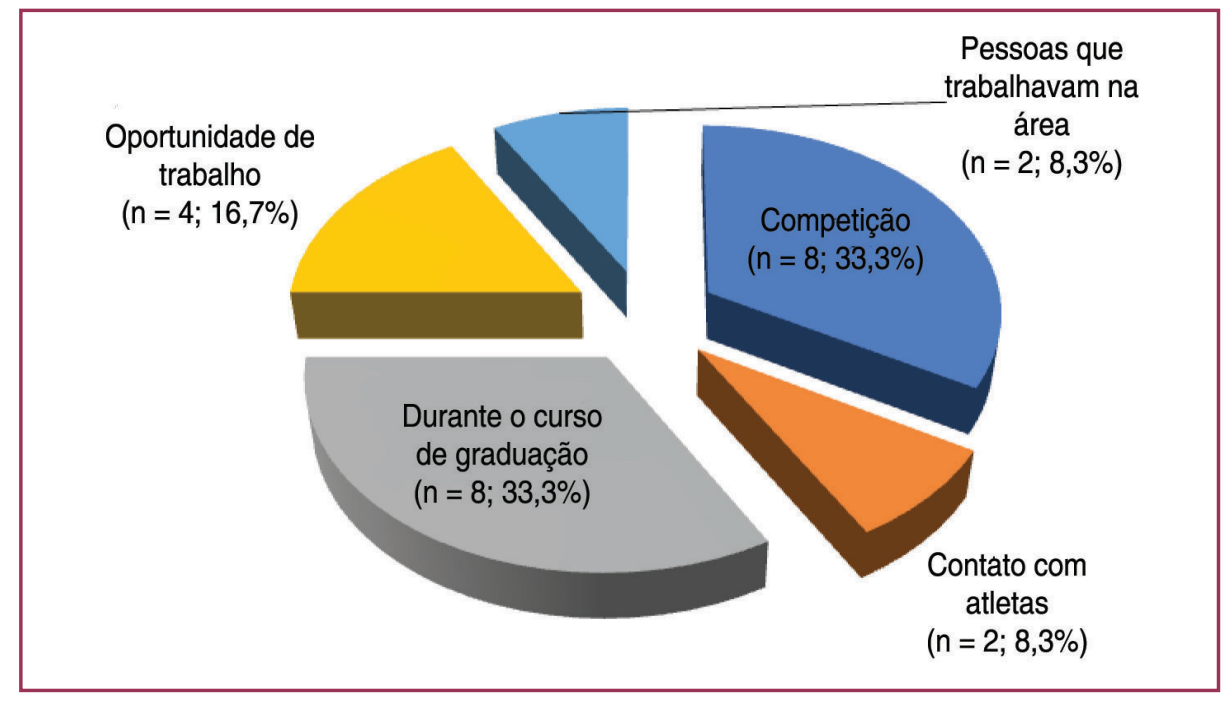

Fonte: Os autores

Em relação aos cursos de pós-graduação, verificamos que $65,7 \%(n=23)$ concluíram cursos de especialização, enquanto $22,9 \%(n=8)$ dos treinadores finalizaram cursos de mestrado em diferentes linhas de pesquisa (Tabela 3). 
Tabela 2 - Disciplinas cursadas pelos treinadores durante a graduação e modalidades esportivas nas quais eles realizaram estágio.

\begin{tabular}{lcc|lcc}
\hline Disciplinas de graduação & N & $\%$ & Estágios & N & $\%$ \\
\hline Educação Física Adaptada & 12 & $44 \%$ & Natação & 7 & $29 \%$ \\
Esporte e Deficiência & 1 & $4 \%$ & Atletismo & 5 & $21 \%$ \\
Atividade Física para Pessoas com Necessidades Especiais & 1 & $4 \%$ & Goalball & 4 & $17 \%$ \\
Atividade Motora Adaptada & 2 & $7 \%$ & Futebol de 5 & 1 & $4 \%$ \\
Atividades Adaptadas & 1 & $4 \%$ & RCR & 2 & $8 \%$ \\
Esporte Adaptado & 5 & $19 \%$ & Bocha & 1 & $4 \%$ \\
Atividade Física Adaptada & 1 & $4 \%$ & Canoagem & 1 & $4 \%$ \\
Esportes Paralímpicos & 1 & $4 \%$ & BCR & 1 & $4 \%$ \\
Atividade Motora para Grupos Especiais & 1 & $4 \%$ & ECR & 1 & $4 \%$ \\
Educação Física Escolar Especial & 1 & $4 \%$ & HCR & 1 & $4 \%$ \\
Educação Física Escolar Adaptada & 1 & $4 \%$ & & & \\
\hline Total & $\mathbf{2 7}$ & $100 \%$ & Total & 24 & $100 \%$ \\
\hline
\end{tabular}

Fonte: Os autores

Legenda: $\mathrm{RCR}=$ Rugby em cadeira de rodas; $\mathrm{BCR}=$ Basquete em cadeira de rodas; $\mathrm{ECR}$ = Esgrima em cadeira de rodas; $\mathrm{HCR}=$ Handebol em cadeira de rodas.

Tabela 3 - Cursos de pós-graduação e linhas de pesquisas cursadas pelos treinadores

\begin{tabular}{|c|c|c|c|}
\hline & Cursos & $\mathrm{N}$ & $\%$ \\
\hline \multirow{14}{*}{$\begin{array}{l}\text { Cursos de } \\
\text { Especialização } \\
\text { (lato sensu) }\end{array}$} & Fisiologia do Exercício & 5 & $21,7 \%$ \\
\hline & Treinamento Desportivo & 4 & $17,3 \%$ \\
\hline & Educação Especial & 2 & $8,7 \%$ \\
\hline & Natação e Atividades Aquáticas & 2 & $8,7 \%$ \\
\hline & Atividade Motora Adaptada & 2 & $8,7 \%$ \\
\hline & Avaliação e Prescrição de Exercícios para Diferentes Populações & 1 & $4,3 \%$ \\
\hline & Preparação Física & 1 & $4,3 \%$ \\
\hline & Voleibol & 1 & $4,3 \%$ \\
\hline & Gestão e Organização em Eventos Desportivos & 1 & $4,3 \%$ \\
\hline & Exercício Físico Aplicado a Grupos Especiais & 1 & $4,3 \%$ \\
\hline & Aspectos Psicológicos da Motricidade Humana & 1 & $4,3 \%$ \\
\hline & Biomecânica & 1 & $4,3 \%$ \\
\hline & Reabilitação & 1 & $4,3 \%$ \\
\hline & Total & 23 & $100 \%$ \\
\hline \multirow{9}{*}{$\begin{array}{l}\text { Linhas de } \\
\text { pesquisa dos } \\
\text { Cursos de } \\
\text { Mestrado (strictu } \\
\text { sensu) }\end{array}$} & Linhas de pesquisa & $N$ & $\%$ \\
\hline & Atividade Física Adaptada & 2 & $25 \%$ \\
\hline & Ciências da Saúde & 1 & $12,5 \%$ \\
\hline & Formação Docente e Práticas Educativas & 1 & $12,5 \%$ \\
\hline & Pedagogia do Movimento & 1 & $12,5 \%$ \\
\hline & Biodinâmica e Desempenho Humano & 1 & $12,5 \%$ \\
\hline & Educação Inclusiva & 1 & $12,5 \%$ \\
\hline & Fisiologia e Bioquímica do exercício & 1 & $12,5 \%$ \\
\hline & Total & 8 & $100 \%$ \\
\hline
\end{tabular}

Fonte: Os autores 
Verificamos que $85 \%(n=30)$ dos treinadores realizaram cursos de formação complementar (Tabela 4), mas 40\% ( $n=12)$ desses treinadores não especificaram qual curso foi realizado. Vale destacar que a maioria dos cursos realizados $(n=12 ; 67 \%)$ foram oferecidos pelo Comitê Paralímpico Brasileiro.

Tabela 4 - Cursos de formação complementar realizados pelos participantes do estudo.

\begin{tabular}{llcc}
\hline Organização & Cursos & $\mathbf{N}$ & $\%$ \\
\hline CPB & Cursos de capacitação do Comitê Paralímpico Brasileiro & 12 & $66,7 \%$ \\
IPC & Cursos de capacitação do Comitê Paralímpico Internacional & 1 & $5,6 \%$ \\
- & Ergonomia e Classes Funcionais & 1 & $5,6 \%$ \\
COB & Curso para treinador do Comitê Olímpico Brasileiro & 1 & $5,6 \%$ \\
- & Educação Física Inclusiva & 1 & $5,6 \%$ \\
- & Treinamento em Paraciclismo & 1 & $5,6 \%$ \\
COB & Curso de gestão do Comitê Olímpico Brasileiro & 1 & $5,6 \%$ \\
\hline & Total & $\mathbf{1 8}$ & $\mathbf{1 0 0 \%}$ \\
\hline
\end{tabular}

Fonte: Os autores

Legenda: $\mathrm{CPB}=$ Comitê Paralímpico Brasileiro, IPC = International Paralympic Committee, $\mathrm{COB}=$ Comitê Olímpico Brasileiro.

Em relação às funções exercidas pelos treinadores no ambiente esportivo, 71,4\% ( $n=$ 25) realizam somente a função de treinador, enquanto $28,6 \%(n=10)$ ocupam também funções de coordenador de modalidades, preparador físico e/ou fisiologista.

\section{DISCUSSÃO}

O objetivo do presente estudo foi analisar a trajetória profissional dos treinadores da delegação brasileira que participaram dos Jogos Paralímpicos Rio 2016. Os resultados do presente estudo corroboram outros estudos reportados na literatura, principalmente pelas diferentes experiências que contribuíram para a trajetória do treinador (CREGAN; BLOOM; REID, 2007; MCMASTER; CULVER; WERTHNER, 2012; DUARTE; CULVER, 2014; TAYLOR; WERTHNER, CULVER, 2014). O ponto chave dessa questão é que os treinadores realizaram cursos de graduação e de formação complementar que não ofereceram informações específicas sobre o processo de treinamento de pessoas com deficiência. Adicionalmente, o aprendizado por meio de fontes informais é considerado como relevante para o desenvolvimento da carreira nessa área (CREGAN; BLOOM; REID, 2007; MCMASTER; CULVER; WERTHNER, 2012; DUARTE; CULVER, 2014; TAYLOR; WERTHNER, CULVER, 2014).

No presente estudo podemos observar que $97,1 \%$ dos treinadores graduaram-se em Educação Física. A formação em Educação Física é considerada fundamental para treinadores, uma vez que oferece conteúdos relacionados a diferentes ciências do esporte (TAYLOR; WERTHNER, CULVER, 2014). No Brasil, somente a partir do ano de 1987 os cursos de Educação Física foram obrigados a inserir em sua grade curricular disciplinas que abordassem as questões relativas às pessoas com deficiência (SILVA, SEABRA JÚNIOR; ARAÚJO, 2008; SIMIM, 2014). Efetivamente, essas disciplinas foram implementadas em 1992, o que prejudicou a participação dos profissionais em tais disciplinas (SIMIM, 2014). Apesar disso, nosso estudo indica que esse fato não foi decisivo para que os treinadores se envolvessem com o esporte 
paralímpico, uma vez que o primeiro contato de $33,3 \%$ dos profissionais com essa temática foi na universidade (Figura 1).

O fato de que $34 \%$ dos treinadores não realizaram disciplinas com a temática da atividade física para pessoas com deficiência não limitou 0 envolvimento desses com a temática do esporte para pessoas com deficiência. Ressaltamos que conhecimentos específicos a respeito das deficiências são necessários para efetividade no trabalho (CÔTÉ; GILBERT, 2009) e desenvolvimento do conhecimento profissional do treinador (CREGAN; BLOOM; REID, 2007; MCMASTER; CULVER; WERTHNER, 2012; TAWSE et al., 2012). Por outro lado, estudos reportam que a trajetória do treinador não se limita à situações formais, pois os cursos de graduação não aprofundam a abordagem de questões sobre atletas com deficiência e suas particularidades no treinamento (DUARTE; CULVER, 2014; FAIRHURST; BLOOM; HARVEY, 2017; WAREHAM et al., 2017; DOUGLAS; FALCÃO; BLOOM, 2018). Para tanto, a aprendizagem ocorre durante toda a vida e até mesmo antes de iniciar o curso superior (DUARTE; CULVER, 2014; MILISTETD et al., 2015). A esse respeito, estudos confirmaram a relevância do contato prévio com pessoas com deficiência antes da formação universitária formal, como forma de aprendizagem e motivação para atuar com este público (DUARTE; CULVER, 2014; TAYLOR; WERTHNER; CULVER, 2014).

Situações informais, como estágios em modalidades esportivas, propiciam o contato com atletas e seus familiares, bem como relações com outros profissionais envolvidos no esporte paralímpico (Tabela 2). Essas atividades são retratadas na literatura como essenciais para que os treinadores adquiriram conhecimentos específicos a respeito do paradesporto (CREGAN; BLOOM; REID, 2007; MCMASTER; CULVER; WERTHNER, 2012; DUARTE; CULVER, 2014; TAYLOR; WERTHNER; CULVER, 2014). Estágios são oportunidades de aprendizado informal por meio de reflexão e interpretação de contextos propostos e orientados por treinadores mais experientes e mentores (MESQUITA et al., 2014). Wareham e colaboradores (2017) destacaram que interação com profissionais mais experientes contribui para aquisição de conhecimentos do processo de treinamento de atletas com deficiência e no desenvolvimento de habilidades de comunicação com esses atletas. $O$ estudo de Duarte e Culver (2014) destacou a relevância de se ter orientadores e/ou mentores na trajetória e formação profissional do treinador. Esses autores ressaltaram que o contato com mentores e/ou treinadores mais experientes é fundamental, principalmente no início da carreira. Os autores apontaram que mentores podem auxiliar e orientar novos treinadores na organização de sessões de treinamento para atletas com deficiência. A organização e planejamento do treinamento é uma das principais funções do treinador (CÔTÉ et al., 1995), e o domínio desse conhecimento é essencial para sua efetividade (CÔTÉ; GILBERT, 2009). Assim, é necessário que programas de capacitação se estruturem para oferecer oportunidades de estágios aos treinadores aspirantes no desenvolvimento de suas habilidades e carreira profissional (FAIRHURST; BLOOM; HARVEY, 2017; WAREHAM et al., 2017; DOUGLAS; FALCÃO; BLOOM, 2018).

Dentro dessa temática, o contato com os pais ou cuidadores dos atletas durante os estágios também se manifesta como estratégia para a aquisição de conhecimentos a respeito do contexto geral da deficiência dos indivíduos no seu dia a dia (DUARTE; CULVER, 2014). Pais ou cuidadores conseguem oferecer perspectivas pertinentes acerca da necessidade dos atletas sobre adaptações necessárias nos seus equipamentos, assim como informações dos medicamentos utilizados pelos atletas (CREGAN; BLOOM; REID, 2007). Do mesmo modo, a 
interação com os atletas oportuniza situações de aprendizado e reflexão, pois as diferentes deficiências necessitam de adaptações específicas para a prática (FALCÃO; BLOOM; LOUGHEAD, 2015; DOUGLAS; FALCÃO; BLOOM, 2018). Para tanto, as especificidades de cada deficiência exigem do treinador habilidades e criação de diferentes estratégias para o processo de treinamento dos paratletas (CREGAN; BLOOM; REID, 2007; DUARTE; CULVER, 2014). Em síntese, essas situações de aprendizado informais conseguem ser mais contextualizadas quando comparadas com as disciplinas e atividades realizadas na maioria dos cursos de graduação (MCMASTER; CULVER; WERTHNER, 2012; DOUGLAS; FALCÃO; BLOOM, 2018).

Em relação à formação continuada (Tabela 3), os treinadores participantes do estudo realizaram cursos de pós-graduação, principalmente de especialização lato sensu. Os cursos de especialização têm o objetivo de aprofundar conhecimentos em relação à atuação com pessoas com deficiência (FILUS; MARTINS JUNIOR, 2004), enquanto cursos de pós-graduação stricto sensu têm por objetivo a produção científica e a formação de professores. Para além dessas questões, cursos de formação complementar (Tabela 4) são meios de oferecer aos treinadores conteúdos específicos desse campo de atuação (WRIGHT; TRUDEL; CULVER, 2007; MCMASTER; CULVER; WERTHNER, 2012; MACDONALD et al., 2016; DOUGLAS; FALCÃO; BLOOM, 2018), como, por exemplo, cursos de modalidades paralímpicas oferecidos pelo Comitê Paralímpico Brasileiro (CPB). Para além dessas questões, a efetividade desses cursos deve ser avaliada de acordo com o nível de experiência do treinador, pois estes apresentam distintas demandas de aprendizagem (WRIGHT; TRUDEL; CULVER, 2007; TRUDEL; CULVER; WERTHNER et al., 2013; TRUDEL; GILBERT, 2013). Nesse contexto, a oferta de cursos em diferentes níveis de aprofundamento auxilia na formação dos treinadores.

Outro ponto relevante a ser discutido concentra-se nas funções exercidas pelo treinador no contexto esportivo. Neste ponto, os treinadores participantes do presente estudo exerceram apenas a função de treinador. Genericamente, as funções dos treinadores consistem na organização e planejamento de sessões treinamento, além de oferecer auxílio aos seus atletas para alcançarem seus objetivos durante as competições (CÔTÉ et al., 1995). Vale ressaltar que os conhecimentos aplicados do treinador em associação com as metas dos atletas são dependentes do nível de competitividade e modalidade em que os esportistas se encontram (CÔTÉ et al., 1995; CÔTÉ; GILBERT, 2009). Contrariamente, a literatura específica reporta que treinadores no esporte paralímpico realizam diferentes tarefas (nutrição, fisiologia, biomecânica, preparação física, psicologia, dentre outras) no corpo técnico das modalidades, principalmente por falta de recursos e particularidades do paradesporto (TAWSE et al., 2012; TAYLOR; WERTHNER; CULVER, 2014). A realização de diferentes tarefas afeta a atuação profissional do treinador, influenciando sua eficiência e ampliando sua necessidade de conhecimento multidisciplinar ao longo da sua trajetória (MESQUITA, 2000). Para tanto, é fundamental que o treinador amplie seus conhecimentos, busque cursos de formação e aperfeiçoamento, interação constante com profissionais da área específica, reflexões durante a prática com seus atletas e constante comunicação com os familiares para aprimorar sua atuação profissional.

Portanto, este estudo buscou explorar a formação de treinadores que atuam no mais alto nível do esporte adaptado, que são os Jogos Paralímpicos. Por serem profissionais que se encontram neste patamar, os resultados do presente estudo não podem ser generalizados aos treinadores que atuam junto a atletas e instituições com menos recursos financeiros e 
escassa infraestrutura, constituindo, assim, fator limitante do presente estudo. Além disso, fazse necessária a análise da formação de treinadores que atuam em outras modalidades para pessoas com deficiência e que não fazem parte do programa paralímpico.

\section{CONCLUSÕES}

Podemos concluir que os treinadores da Delegação Paralímpica Brasileira que atuaram durante os Jogos Paralímpicos Rio 2016 tiveram ao longo da sua trajetória profissional oportunidades de aprendizado formal por meio de cursos de graduação e pós-graduação, além de cursos informais complementares, os quais foram fundamentais para construir a trajetória profissional desses treinadores. Apesar da pluralidade das experiências vividas pelos treinadores, 42,9\% desses realizaram estágios supervisionados em Esportes Paralímpicos durante a graduação em Educação Física, sendo considerados vitais para o desenvolvimento do conhecimento e da carreira desses profissionais. Nesta perspectiva, podemos sugerir e oferecer disciplinas de estágio em Atividade Física Adaptada ou Esportes Paralímpicos, bem como estabelecer parcerias com associações e/ou clubes que trabalham com pessoas e com atletas com deficiência, para que os alunos possam ter essa vivência durante sua graduação.

\section{REFERÊNCIAS}

CÔTÉ, Jean; GILBERT, Wade. An Integrative Definition of Coaching Effectiveness and Expertise. International Journal of Sports Science \& Coaching, v. 4, n. 3, p. 307-323, 2009. Disponível em: http://journals.sagepub.com/doi/10.1260/174795409789623892. Acesso em: 25 ago. 2018.

CÔTÉ, Jean et al. The Coaching Model: A Grounded Assessment of Expert Gymnastic Coaches' Knowledge. Journal of Sport \& Exercise Psychology, v. 17, n. 1, 1995. Disponível em: https:// journals.humankinetics.com/doi/10.1123/jsep.17.1.1. Acesso em: 25 ago. 2018.

CREGAN, Kerry; BLOOM, Gordon A.; REID, Greg. Career Evolution and Knowledge of Elite Coaches of Swimmers With a Physical Disability. Research Quarterly for Exercise and Sport, v. 78, n. 4, p. 339-350, 2007. Disponível em: https://www.tandfonline.com/doi/abs/10.1080/0270136 7.2007.10599431. Acesso em: 25 ago. 2018.

DOUGLAS, Scott; FALCÃO, William. R.; BLOOM, Gordon. A. Career Development and Learning Pathways of Paralympic Coaches With a Disability. Adapted Physical Activity Quartely, v. 35, n. 1, p. 93-110, 2018. Disponível em: http://dx.doi.org/10.1123/apaq.2017-0010. Acesso em: 25 ago. 2018.

DUARTE, Tiago; CULVER, Diane M. Becoming a Coach in Developmental Adaptive Sailing: A Lifelong Learning Perspective. Journal of Appled Sport Psychology, v.26, 2014. p.441-56. Disponível em: https://www.ncbi.nlm.nih.gov/pmc/articles/PMC4151822/. Acesso em 25 ago. 2018.

ERICSSON, K. Anders. Deliberate practice and acquisition of expert performance: a general overview. Academic Emergency Medicine, v. 15, n. 11, p.988 - 994. 2008. Disponível em: https://doi.org/10.1111/j.1553-2712.2008.00227.x. Acesso em 29 jan. 2019.

ERICSSON, K. Anders. Expertise. Current Biology, v. 24, n. 11, p. R508-R510, 2014. 
FAIRHURST, Katherine E.; BLOOM, Gordon A.; HARVEY, William J. The learning and mentoring experiences of Paralympic coaches. Disability and Health Journal, v. 10, n. 2, p. 240-246, 2017. Disponível em: http://www.sciencedirect.com/science/article/pii/S1936657416301431. Acesso em: 25 ago. 2018.

FALCÃO, William R.; BLOOM, Gordon A.; LOUGHEAD, Todd M. Coaches' Perceptions of Team Cohesion in Paralympic Sports. Adapted Physical Activity Quarterly, v. 32, p. 206-222, 2015. Disponível em: https://journals.humankinetics.com/doi/10.1123/APAQ.2014-0122. Acesso em: 25 ago. 2018.

FILUS, Josian F.; MARTINS JUNIOR, Joaquim. Reflexões sobre a formaç̧ão em Educação Física e a sua aplicação no trabalho junto às pessoas com deficiência. Revista da Educação Física/ UEM, v. 15, n. 2, p. 79-87, 2004. Disponível em: http://periodicos.uem.br/ojs/index.php/ RevEducFis/article/view/3424. Acesso em: 25 ago. 2018.

MACDONALD, Dany J. et al. Understanding Sources of Knowledge for Coaches of Athletes with Intellectual Disabilities. Journal of Applied Research in Intellectual Disabilities, v. 29, n. 3, p. 242-249, 2016. Disponível em: https://onlinelibrary.wiley.com/doi/abs/10.1111/jar.12174. Acesso em: 25 ago. 2018.

MCMASTER, Sarah; CULVER, Diane; WERTHNER, Penny. Coaches of athletes with a physical disability: a look at their learning experiences. Qualitative Research in Sport, Exercise and Health, v. 4, n. 2, p. 226-243, 2012. Disponível em: https://www.tandfonline.com/doi/ abs/10.1080/2159676X.2012.686060?journalCode=rqrs21. Acesso em: 25 ago. 2018.

MESQUITA, Isabel et al. Coach Learning and Coach Education: Portuguese Expert Coaches' Perspective. The Sport Psychologist, v. 28, n. 2, p. 124-136, 2014. Disponível em: https:// journals.humankinetics.com/doi/abs/10.1123/tsp.2011-0117. Acesso em: 25 ago. 2018.

MESQUITA, Isabel. A Pedagogia do Treino: a formação em jogos desportivos coletivos. 2. ed. Lisboa: Livros Horizonte, 2000.

MILISTETD, Michel et al. A Aprendizagem Profissional de Treinadores Esportivos: Desafios da Formação Inicial Universitária em Educação Física. Pensar a Prática, v. 18, n. 4, p. 982-994, 2015. Disponível em: https://www.revistas.ufg.br/fef/article/view/34988. Acesso em: 25 ago. 2018.

PERRET, Claudio. Elite-adapted wheelchair sports performance: a systematic review. Disability and Rehabilitation, v. 39, n. 2, p. 164-172, 2015. Disponível em: https://www.tandfonline.com/doi/ abs/10.3109/09638288.2015.1095951. Acesso em: 25 ago. 2018.

SILVA, Rita de Fátima da; SEABRA JÚNIOR, Luiz; ARAÚJO, Paulo Ferreira de. Educação física adaptada no Brasil: da história à inclusão educacional. São Paulo: Phorte, 2008.

SIMIM, Mário Antônio de Moura. Exercício, Esporte e Inclusão: a formação do profissional de educação física e o esporte adaptado. In: NOCE, F. O Profissional de Educação Física na Área da Saúde. Belo Horizonte: EEFFTO, 2014. p. 71-80.

TAWSE, Holly et al. The role of coaches of wheelchair rugby in the development of athletes with a spinal cord injury. Qualitative Research in Sport, Exercise and Health, v. 4, n. 2, p. 206-225, 2012. Disponível em: https://www.tandfonline.com/doi/abs/10.1080/2159676X.2012.685104. Acesso em: 25 ago. 2018.

TAYLOR, Shaunna L.; WERTHNER, Penny; CULVER, Diane. A Case Study of a Parasport Coach and a Life of Learning. International Sport Coaching Journal, v. 1, p. 127-138, 2014. 
Disponível em: https://journals.humankinetics.com/doi/abs/10.1123/iscj.2013-0005. Acesso em: 25 ago. 2018.

TRUDEL, Pierre; CULVER, Diane; WERTHNER, Penny. Looking at coach development from the coach-learner's perspective: Considerations for coach development administrators. In: POTRAC, P.;GILBERT, Wade, et al (ed.). Routledge Handbook of Sports Coaching. New York: Routledge, 2013. p. 375-387.

TRUDEL, Pierre; GILBERT, Wade. The Role of Deliberate Practice in Becoming an Expert Coach: Part 3 - Creating Optimal Settings. Olympic Coach Magazine, v. 24, n. 2, 2013.

VOLPATO, Gilson. Dicas de redação científica. 4. ed. Botucatu: Best Writing, 2016.

WAREHAM, Yvette et al. Coaching athletes with disability: preconceptions and reality. Sport in Society, v. 20, n. 9, p. 1185-1202, 2017. Disponível em: https://www.tandfonline.com/doi/ abs/10.1080/17430437.2016.1269084?journalCode=fcss20. Acesso em: 25 ago. 2018.

WRIGHT, Trevor; TRUDEL, Pierre; CULVER, Diane. Learning how to coach: the different learning situations reported by youth ice hockey coaches. Physical Education and Sport Pedagogy, v. 12, n. 2, p. 127-144, 2007. Disponível em: https://www.tandfonline.com/doi/ref/10.10 80/17408980701282019?scroll=top. Acesso em: 25 ago. 2018.

\section{Agradecimentos:}

Os autores agradecem ao Centro de Estudos em Psicobiologia e Exercício (CEPE), o Centro Multidisciplinar em Sonolência e Acidentes (CEMSA), a Fundação de Amparo à Pesquisa do Estado de Minas Gerais (FAPEMIG), a Coordenação de Aperfeiçoamento de Pessoal de Nível Superior (CAPES), o Conselho Nacional de Desenvolvimento Científico e Tecnológico (CNPQ) e a Academia Paralímpica Brasileira do Comitê Paralímpico Brasileiro. 